\title{
DYNAMIC RADIO RESOURCE MANAGEMENT IN GSM/GPRS USING SCALABLE RESOURCE ALLOCATION TECHNIQUE
}

\author{
Seok Y Tang, Shyamalie Thilakawardana and Rahim Tafazolli \\ Mobile Communications Research Group \\ Centre for Communications System Research, University of Surrey, U.K \\ seok.tang@eim.surrey.ac.uk
}

\begin{abstract}
Scalable Resource Allocation (ScRA) algorithm is developed to improve the mobile network radio resource utilization [1]. The traditional mobile dimensioning is based on the "busy hour" traffic intensity, and this Static Resource Allocation (StRA) methodology does not seem to be able to provide efficient radio resource utilization for the future/present multi services environment, with their expected spatially and temporally varying loads. This is in hindrance for the introduction of wireless IP based services, for which the demand is rapidly increasing.
\end{abstract}

This paper provides an extension analysis by incorporating single slot FIFO and single slot Round Robin (single slot $\mathrm{RR})$, blocked-call cleared (BCC) and blocked-call delayed (BCD) strategies in the ScRA scheme. By employing the ScRA scheme in an example GSM and GPRS network, we specifically investigate and evaluate the system throughput for both the circuit-and packet-switched networks.

The findings show that single slot FIFO ScRA and single slot RR ScRA schemes obtained no difference in system throughput. On the other hand, when BCD is implemented in ScRA scheme, there is significant throughput gain.

Keywords - scalable resource allocation, scheduling operations, blocked-calls delayed, GPRS

\section{INTRODUCTION}

With the possibility that there is a lack of radio resource to support continuous increase of wireless services, recently, regulators are rethinking new strategies for spectrum allocation. In fact, recent research efforts have been focused on dynamically allocating spectrum between two radio access networks (RANs) for Universal Mobile Telecommunications Systems (UMTS) and Digital Video Broadcasting Terrestrial (DVB-T) [2], [3], [4], [5].

ScRA scheme borrowed the idea of dynamically allocating radio resource between two access networks. The primary aim is to better cope with the non-uniform traffic characteristics of GSM/GPRS network. Particularly, we focus in radio resource management for the downlink traffic, which comprises multiple services.

Currently, the integrated GSM and GPRS network share a common spectrum whereby each cell is allocated a pool of channels. Under the StRA scheme, channels allocated to the GSM network is based on the busy hour traffic intensity, leaving the remainder pool of channels allocated permanently to the GPRS network [1]. Therefore, independent of the load the number of voice channel is fixed and predetermined throughout the resource allocation. Hence, drawback of this scheme is that this channel assignment does not adapt to the time-varying voice service and much of the scarce resource in the GSM system is not used efficiently, or even left unused, especially during the non-peak hours. On the other hand at this moment, the GPRS system may be in desperate need of radio resource to support the ever-increasing data connections.

In line with the future demand driven resource allocation, ScRA scheme is proposed [1] to better utilize the radio resource and hence support more multiple and diverse services in the future mobile environment. ScRA scheme allocates the common resources dynamically and periodically between the GSM and GPRS networks. Indeed, simulation results showed that the ScRA scheme achieves greater radio resource utilization over StRA scheme whilst still providing desirable QoSs [1]. This paper provides an extension analysis in the ScRA scheme. Different scheduling techniques and blocked-calls strategies are incorporated in the ScRA scheme. The focus of this paper is to evaluate the performance difference in terms of throughput when four strategies, namely single slot FIFO, single slot $\mathrm{RR}, \mathrm{BCC}, \mathrm{BCD}$, are to be incorporated into the ScRA scheme.

The rest of the paper is organized as follows: Section II presents the details of our simulation model. Section III describes the ScRA scheme, and section IV elaborates the two scheduling operations and blocked-calls strategies. Next, section $\mathrm{V}$ illustrates the simulation results and discussion. Finally, conclusions are made in Section VI.

\section{SIMULATION MODEL}

We consider a single cell area under GSM and GPRS systems radio coverage. A base transmitter station is assigned with one carrier frequency serving mixed traffic to mobile stations (MS) via TDMA technology. In our simulation, it is assumed all the eight time slots in a TDMA frame carry either circuit-or packet-switched traffic. Each 
physical channel (time slot) in a TDMA frame has duration of $576.9 \mu \mathrm{s}$. For conventional GSM, a time slot is allocated to the voice service for the entire call duration. And for GPRS data transmission, incoming data-packets generated will be segmented into RLC data blocks and in the data-link layer, a physical channel (time slot) will be assigned to a RLC data block for $20 \mathrm{~ms}$. This is because a GPRS basic radio transmission block or RLC/MAC block consists of four time slots in the consecutive frames; which has a holding time of $20 \mathrm{~ms}$. The sequence of how each user RLC blocks are to be transmitted on the physical channels is determined by the scheduling technique employed. The scheduling techniques used in our simulator will be discussed in section IV.

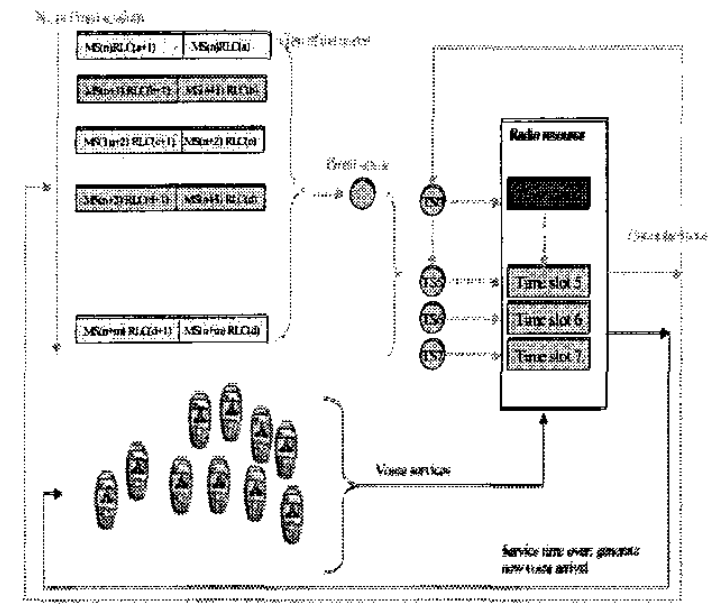

Figure 1: Illustration of mixed traffic environment served by a single carrier

In this mixed traffic environment, two traffic models are used. Email traffic model is based on the statistics collected on Email usage from Finnish University and Research Network, namely FUNET model [6]. The probability distribution function $f(\chi: \alpha, \beta)$ of the Email connection sizes is given by

$$
f(x, \alpha, \beta)=\frac{\beta}{\pi\left(\beta+(x-\alpha)^{2}\right)}
$$

where it can be approximated by a truncated Cauchy distribution ( $\alpha=0.8, \beta=1$ ) with a maximum message size of 10 Kbytes and average size of the Cauchy Email connection is 830 bytes [6]. We have assumed that voice calls and Email sessions arrival as Poisson distribution process. On the hand, voice service holding time is Exponential distributed with three sets of holding time; i.e. $90 s, 120 \mathrm{~s}$ and $250 \mathrm{~s}$. The probability density function of the Exponential distribution with a mean value of $(1 / \mu)$ is expressed as below,

$$
f(x, \mu)=\mu e^{-\mu x}
$$

As mentioned earlier, ScRA scheme is employed to allocate the radio resources between circuit-and packet-switched services periodically.

\section{DESCRIPTION OF SCALABLE RESOURCE ALLOCATION}

Each Email session arrives at GPRS Base Station System (BSS) with independent inter-arrival times and these interarrival times for every Email user is identically Exponentially distributed.

\section{A. Single -slot RR scheduling technique}

As shown in Figure 2, a newly generated Email session will join the shortest queue and each time slot (resource) has separate queue. In case of ties, this Email session will choose the shortest queue from the left. In single slot RR scheduling technique, there is a central controller in each time slot queue. This controller is responsible for permitting users to access to the shared time slot.

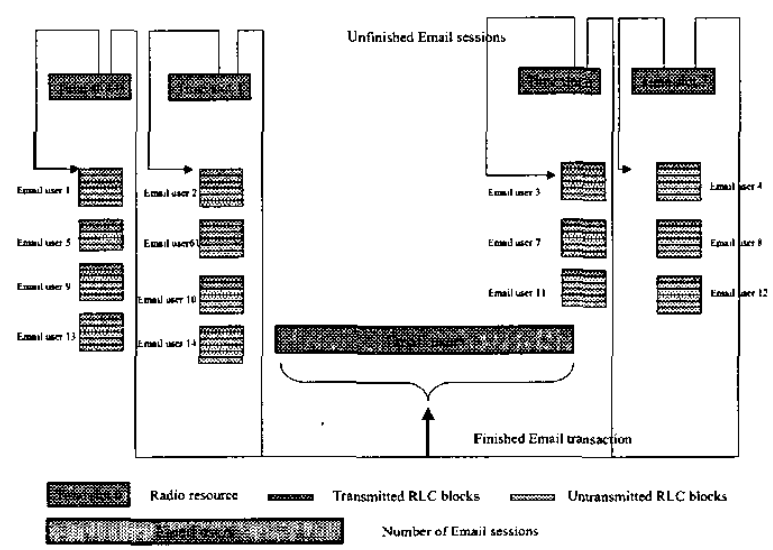

Figure 2: Single slot RR scheduling concept

For example, this central controller will issue messages to the Email users in the time slot queue so that each user can use the time slot for $20 \mathrm{~ms}$ (a RLC block transmission time) for each round. An incomplete Email transaction/user will return back to the time slot queue; and will wait for its tum to transmit on the time slot again. Finally if the user has finished its Email transaction, another arrival Email user will be generated into the system. This newly generated user will again join the shortest time slot queue and the process repeats.

As an illustration, time slot 0 is assigned to $\mathrm{N}$ Email users in the queue for data transfer (see Figure 3). Each Email user will be contacted, polled and invited to transmit its RLC block on time slot 0 by the central controller. After each RLC block is transmitted, in every next transmission, different users in the line will be polled by the central controller and will be served. 


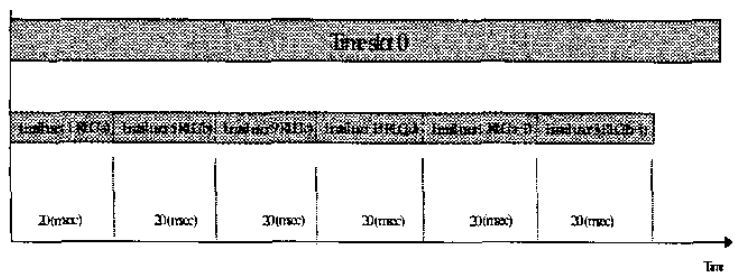

Figure 3: RLC blocks transmission based on single slot RR scheduling techniques

\section{B. Single slot FIFO scheduling technique}

As shown in Figure 4, a newly generated Email session will join a single queue and each time slot (resource) has separate queue. A central controller is responsible for a single user access to the designated time slot till it finishes the complete Email session. Once the time slot had transmitted all the RLC blocks for each user, the central controller perform two operations. First, it will invite the Email user next in line to transmit on the designed time slot and next, it will trigger the traffic generator for another Email arrival.

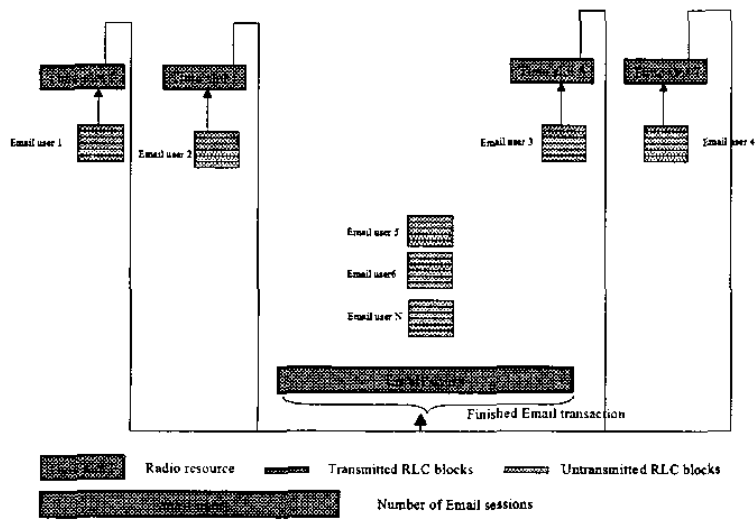

Figure 4: Single slot FIFO scheduling concept

Figure 5 shows an illustration of how the time slot 0 is used for data transfer. It is shown that time slot 0 is assigned to a single Email user for a complete Email session data transfer and Email user is served one after another.
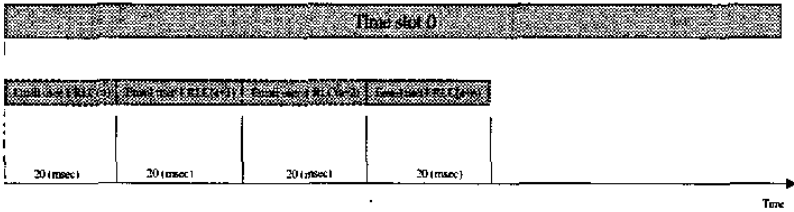

Figure 5: RLC blocks transmission based on single slot FIFO scheduling techniques

Additionally, the Email arrival event is illustrated in Figure 6(a). Each newly generated Email session has its own record of arrival time and total number of RLC blocks needed to be transmitted. This newly arrived Email record is then placed at the end of the queue. If the radio resource (time slot) is free, the function resource_scheduling routine is invoked.

The resource_scheduling event is illustrated in Figure 6(b). When this routine is invoked, the first Email session will be taken out from the time slot queue. The purpose of this routine is to take the first Email job out of the queue, and place this job on the free time-slot.

For single slot FIFO scheduling technique, this Email user will occupy the time slot for entire duration of the session. Once this user is released from the time slot, the next user in line will be served (see Figure 5). However, for single slot RR scheduling technique, every Email user will be served only for one RLC block duration and then another user in line will be served (see Figure 3). After every $20 \mathrm{~ms}$, the RLC block counter for this user will be decremented, and the end_resource_run routine (Figure 6(c)) is invoked. After every RLC block transmission $(20 \mathrm{~ms})$, this RLC block will be removed from the time slot occupation and a check is made to see if this user RLC completes its email transaction (needs more radio resource), i.e. if RLC block counter $>0$, this user will be put back to the time slot queue. Again, resource_scheduling routine is invoked to remove the first job from the time slot queue. On the other hand, if this is the last RLC block for the Email transaction, the response time of this Email transaction and statistics will be gathered. Subsequently, another Email arrival will be scheduled into the system. Eventually, a check is made to see whether the end simulation time has reached; if so, this simulation will end and the performance report will be generated. If the simulation is not over, and if there are Email users in the queue, the resource scheduling routine will again be invoked. On the other hand, if the queue is empty, this time slot will be initialized as idle and the simulation continues.

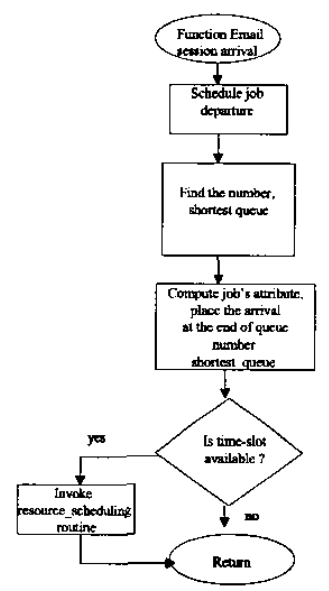

Figure 6 (a): Flowchart for

arrival routine

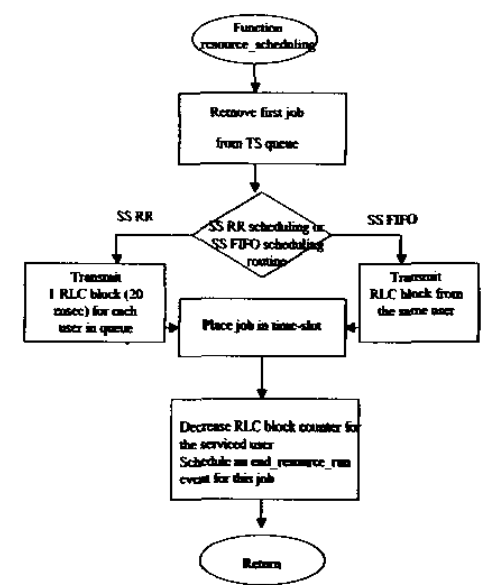

Figure $6(\mathrm{~b})$ : resource scheduling routine 


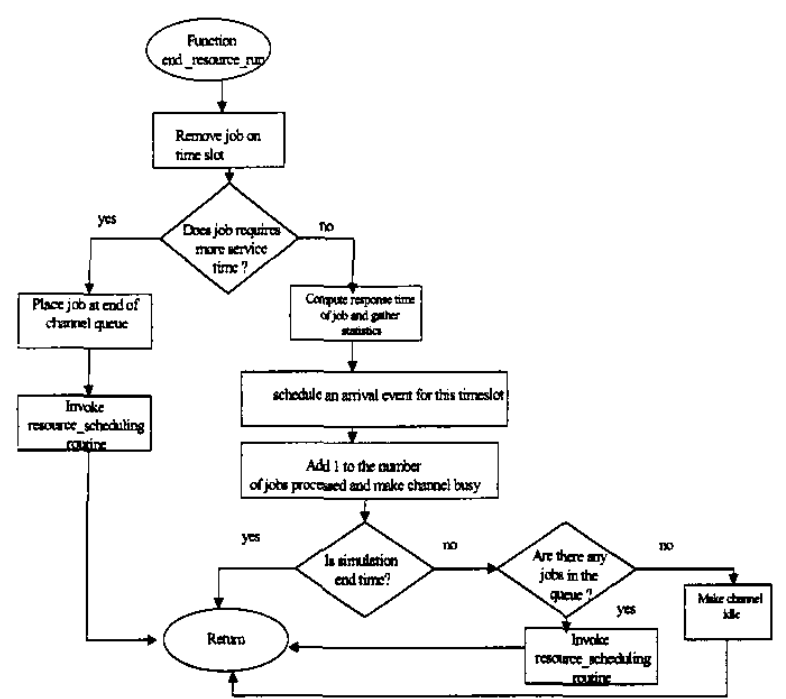

Figure $6(\mathrm{c})$ : Flowchart for end_resource_scheduling routine

In the following, $\mathrm{BCC}$ and $\mathrm{BCD}$ strategies are explained. The $\mathrm{BCD}$ and $\mathrm{BCC}$ strategies are implemented in GSM serving circuit-switched voice service. In GSM, a dedicated communications path is set up between parties. The advantage of circuit-switching is that once a call has been set up, the users of that path can then transfer information unaffected by the load on the network, and this connection is guaranteed for the entire duration of that call.

The voice call arrival is Poisson distributed. It is assumed that there is only one queue in the GSM network when BCD is simulated.

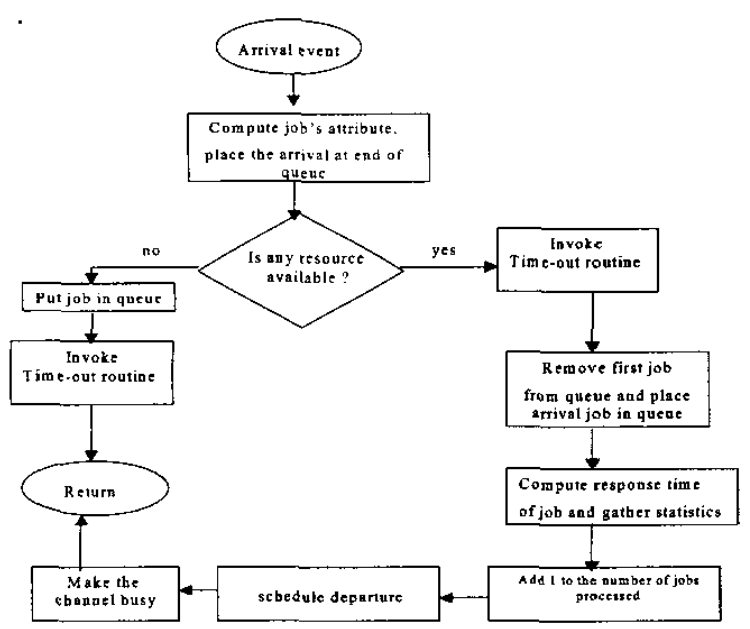

Figure 7 (a) : Flowchart for voice arrival routine

Figure 7(a) flowcharted the BCD strategy arrival routine. The newly arrived voice user with its attributes of arrival time and service time will join the end of the queue. Next, the voice time slot is checked for its status; whether it is busy or idle. If there is no time slot available, the newly arrived voice service will stay in the queue and time-out routine is invoked. In this study, time-out is defined as the time difference when voice service arrived in queue to the simulation time whenever time-out routine is invoked. For this study, it is assumed that the system can tolerate for four sets of time-out timing, i.e. $0 s, 5 s, 10 s, 15 s$.

Basically, the time-out routine will check if there is any time-out voice users in the queue, if there is; the block-call counter will increase in accordance to the number of timeout voice users; else, the simulation continues.

With regards to the BCC strategy, there is no queue to store the voice user if there is no time slot available, the voice user will be denied from the system immediately.

On the other hand, if there is time slot available, the status of the queue will be checked (for BCD); i.e. any jobs in the queue? If there is no previous voice calls in the queue, the newly arrived job will be served by this free time slot; else, the time-out routine will be invoked. This time-out routine checks on the number of voice calls that are time-out and thus triggers the block-call counter to increase accordingly. Subsequently, the time slot will serve the first job in the line and departure of this job will be scheduled.

Upon departure of voice user (see Figure 7 (b)), the queue status is first checked. If there is user in the queue, this user will be placed on the free time slot and consequently this time slot will be initialized as busy, else if there is no voice user in the line, the channel will be initialized as idle.

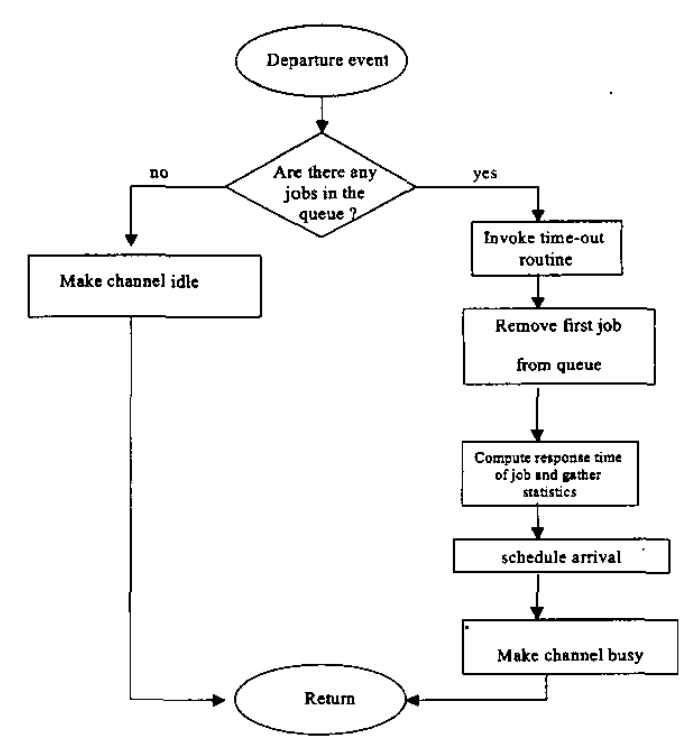

Figure 7 (b) : Flowchart for voice departure routinc 


\section{SIMULATION RESULTS FOR PERFORMANCE COMPARISON}

A. Performance Comparison between single slot FIFO and single slot RR Scheduling Schemes implemented within ScRA scheme

Figure 8 shows the Email throughput results against Email session arrival rate per hour. The simulator is modelled with increasing Email session arrival rate by varying number of Email users from 10 to 10000 . The simulation results illustrate that system throughput increases with increasing Email arrival rate. The result obtained shows there is no significant difference in the system throughput performance between single slot FIFO and single slot RR scheduling techniques.

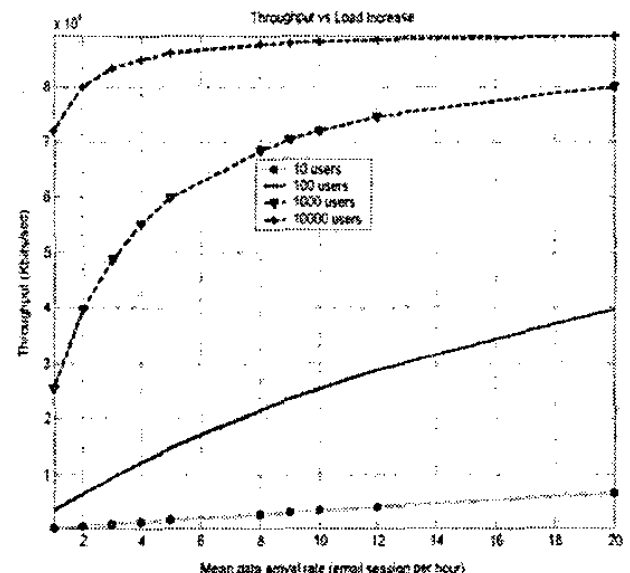

Figure 8: Throughput obtained from single slot FIFO scheduling technique

It is interesting to note that as single slot FIFO and single slot RR techniques obtained no significant difference in system throughput. With regards to the implementation complexity of the scheduling algorithm, it is expected that the processing of single slot FIFO scheduling scheme is less complex than single-slot $\mathrm{RR}$ scheduling scheme. It is because single slot FIFO technique allocates a free timeslot to the Email session solely based on first in first serve manner. During the processing of a user Email session, no other process can have the resource until the first user Email session is completely served. Radio resource will only service next user in line (in queue) upon completion of each Email session. Clearly, single slot RR scheduling technique incurs higher computational overhead in assigning and reassigning the resource to each Email user. Hence, single slot FIFO ScRA implementation is easier than single slot RR ScRA technique. But it comes about at the expense of fairness of service distribution for various users. Also, the simulated results suggested that single slot FIFO scheduling scheme is a better choice than single slot RR scheduling scheme when the load is low. This is because single slot FIFO ScRA scheme has the same throughput as single slot RR ScRA scheme but with much simpler implementation.

B. Performance Comparison between Blocked-Calls Cleared and Blocked-Calls Delayed implemented within ScRA scheme

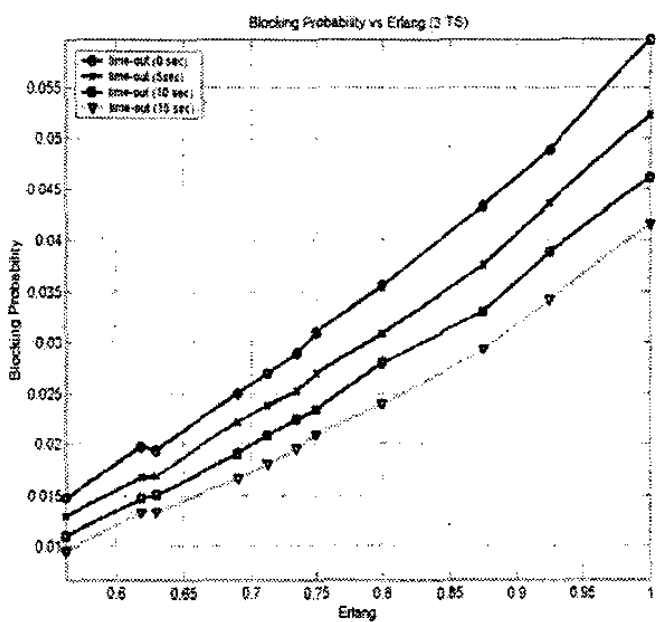

Figure $9(\mathrm{a})$ : Blocking probability performance comparison between $\mathrm{BCD}$ and BCC strategies (holding time $=90 \mathrm{~s}$ )

Table 1 : Holding Time $-90 \mathrm{sec}, 3$ timeslots

\begin{tabular}{|l|l|l|l|l|l|}
\hline $\begin{array}{l}\text { Time-out } \\
\text { (sec) }\end{array}$ & 0 & 5 & 10 & 15 & Remarks \\
\hline $\begin{array}{l}\text { Carried } \\
\text { traffic } \\
\text { difference } \\
\text { (E) }\end{array}$ & 0.64 & 0.67 & 0.70 & 0.74 & $\mathrm{P}_{\mathrm{b}}=2 \%$ \\
\cline { 2 - 6 } & 0.74 & 0.77 & 0.84 & 0.88 & $\mathrm{P}_{\mathrm{b}}=3 \%$ \\
\hline $\begin{array}{l}\text { Percent } \\
\text { increase }\end{array}$ & Reference & 4.47 & 9.38 & 15.6 & $\mathrm{P}_{\mathrm{b}}=2 \%$ \\
\cline { 2 - 6 } & Reference & 4.05 & 12.8 & 18.2 & $\mathbf{P}_{\mathrm{b}}=3 \%$ \\
& & & & & \\
\hline
\end{tabular}




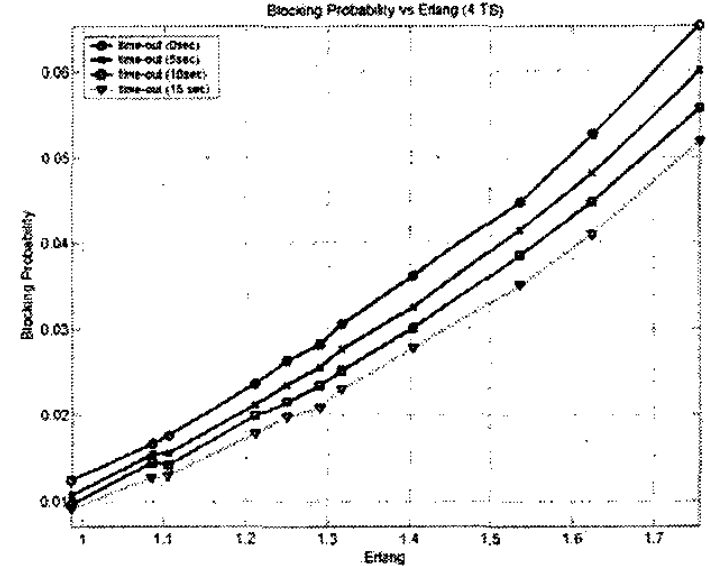

Figure $9(\mathrm{~b})$ : Blocking probability performance comparison between $\mathrm{BCD}$ and $\mathrm{BCC}$ strategies (holding time $=160 \mathrm{~s}$ )

Table 2 : Holding Time $-160 \mathrm{sec}, 4$ timeslots

\begin{tabular}{|l|l|l|l|l|l|}
\hline $\begin{array}{l}\text { Time-out } \\
(\mathrm{sec})\end{array}$ & 0 & 5 & 10 & 15 & Remarks \\
\hline $\begin{array}{l}\text { Carried } \\
\text { traffic } \\
\text { difference } \\
\text { (E) }\end{array}$ & 1.15 & 1.195 & 1.22 & 1.26 & $\mathrm{P}_{\mathrm{b}}=2 \%$ \\
\cline { 2 - 6 } & 1.303 & 1.36 & 1.4 & 1.45 & $\mathrm{P}_{\mathrm{b}}=3 \%$ \\
\hline $\begin{array}{l}\text { Percent } \\
\text { increase }\end{array}$ & Reference & 3.91 & 6.09 & 9.57 & $\mathrm{P}_{\mathrm{b}}=2 \%$ \\
\cline { 2 - 6 } & Reference & 5.7 & 9.7 & 14.7 & $\mathbf{P}_{\mathrm{b}}=3 \%$ \\
\hline
\end{tabular}

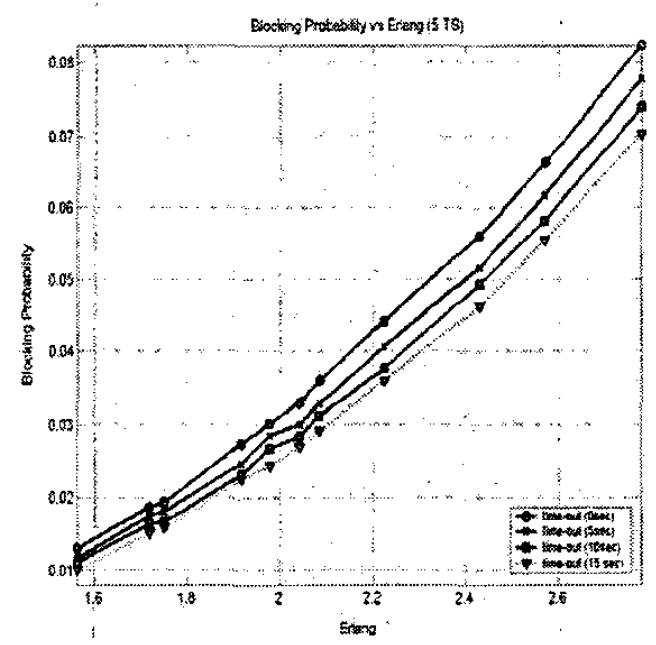

Figure 9(c) : Blocking probability performance comparison between $\mathrm{BCD}$ and $\mathrm{BCC}$ strategies (holding time $=250 \mathrm{~s}$ )
Table 3 : Holding Time $-250 \mathrm{sec}, 5$ timeslots

\begin{tabular}{|l|l|l|l|l|l|}
\hline $\begin{array}{l}\text { Time-oul } \\
(\mathrm{sec})\end{array}$ & 0 & 5 & 10 & 15 & Remarks \\
\hline $\begin{array}{l}\text { Cartied } \\
\text { traffic } \\
\text { difference } \\
(\mathrm{E})\end{array}$ & 1.75 & 1.80 & 1.815 & 1.82 & $\mathrm{P}_{\mathrm{h}}=2 \%$ \\
\cline { 2 - 6 } & 1.97 & 2.07 & 2.09 & 2.10 & $\mathrm{P}_{\mathrm{b}}=3 \%$ \\
\hline $\begin{array}{l}\text { Percent } \\
\text { increase }\end{array}$ & Reference & 2.86 & 3.71 & 3.85 & $\mathrm{P}_{\mathrm{h}}=2 \%$ \\
\cline { 2 - 6 } & Reference & 5.08 & 6.09 & 6.60 & $\mathrm{P}_{\mathrm{b}}=3 \%$ \\
& & & & & \\
\hline
\end{tabular}

\section{CONCLUSION}

The purpose of this research is to provide an insight for the network designer to better utilize the limited radio resources on a dynamic and flexible basis in the multiple services environment.

This study illustrated that when appropriate scheduling technique, and blocked-calls strategies incorporated into the ScRA scheme, the system throughput can be further increased.

\section{REFERENCES}

[1] Tang S, Tafazolli,R "Scalable Resource Allocation Algorithm for GPRS," in IEEE Vehicular Technology Conference, South Korea, Jeyu, Spring 2003.

[2] Sirbu M, Peha $J$ "Spectrum Sharing through Dynamic Channel Assignment for Open Access to Personal Communications Services," in Proceedings of IEEE International Communications Conference (ICC), pp. 417-422, June 1995 .

[3] Leaves P, Ghaheri-Niri S, Tafazolli R, Christodoulides L, Sammut T, Stahl W, Huschke J "Dynamic Spectrum Allocation in a multi-Radio Environment: Concept and Algorithm,"in Second IEE International Conference on $3 G$ Mobile Communication Technologies, London, United Kingdom, pp.53-57, March 2001.

[4] Leaves P, Ghaheri-Niri S, Tafazolli R, Huschke J “ Dynamic Spectrum Allocation in Hybrid Networks with Imperfect Load Prediction," in Third IEE International Conference on $3 G$ Mobile Communication Technologies, London, United Kingdom, pp.444-448, May 2002.

[5] Leaves P, Tafazolli R "A Time Adaptive Dynamic Spectrum Allocation Scheme for a Converged Cellular and Broadcast System," in IEE Getting the Most Out of the Radio Spectrum Conference, London, United Kingdom, pp.444-448, May 2002.

[6] Brasche G, Walke B "Concepts, Services, and Protocols of the New GSM Phase 2+ General Packet Radio Service," in IEEE Communications Magazine, pp.97103, August 1997. 